\title{
The challenges in colorectal cancer management during COVID-19 epidemic
}

\author{
Xianghai Ren ${ }^{1,2,3,4,5,6 \#}$, Baoxiang Chen ${ }^{1,3,4,5,6 \#}$, Yuntian Hong ${ }^{1,3,4,5,6}$, Weicheng Liu $^{1,3,4,5,6}$, Qi Jiang ${ }^{7}$, \\ Jingying Yang ${ }^{8}$, Qun Qian ${ }^{1,3,4,5,6}$, Congqing Jiang ${ }^{1,3,4,5,6}$
}

${ }^{1}$ Department of Colorectal and Anal Surgery, Zhongnan Hospital of Wuhan University, Wuhan 430071, China; ${ }^{2}$ Graduate School of Peking Union Medical College, Chinese Academy of Medical Sciences, Beijing 100730, China; ${ }^{3}$ Clinical Center of Intestinal and Colorectal Diseases of Hubei Province, Wuhan 430071, China; ${ }^{4}$ Hubei Key Laboratory of Intestinal and Colorectal Diseases (Zhongnan Hospital of Wuhan University), Wuhan 430071, China; ${ }^{5}$ Colorectal and Anal Disease Research Center of Medical School (Zhongnan Hospital of Wuhan University), Wuhan 430071, China; ${ }^{6}$ Quality Control Center of Colorectal and Anal Surgery of Health Commission of Hubei Province, Wuhan 430071, China; ${ }^{7}$ Department of Pathology and Pathophysiology, Hubei Provincial Key Laboratory of Developmentally Originated Disease, School of Basic Medical Sciences, Wuhan University, Wuhan 430071, China; ${ }^{8}$ Department of Anesthesia Surgery, Zhongnan Hospital of Wuhan University, Wuhan University, Wuhan 430071, China

Contributions: (I) Conception and design: X Ren, B Chen, C Jiang; (II) Administrative support: Q Qian, C Jiang; (III) Provision of study materials or patients: All authors; (IV) Collection and assembly of data: X Ren, B Chen, C Jiang; (V) Data analysis and interpretation: All authors; (VI) Manuscript writing: All authors; (VII); Final approval of manuscript: All authors.

\#These authors contributed equally to this article.

Correspondence to: Congqing Jiang, MD, PhD. Department of Colorectal and Anal Surgery, Zhongnan Hospital of Wuhan University, Wuhan 430071, China. Email: wb002554@whu.edu.cn; Qun Qian, MD, PhD. Department of Colorectal and Anal Surgery, Zhongnan Hospital of Wuhan University, Wuhan 430071, China. Email: qunqian2007@163.com.

\begin{abstract}
It has been over 2 months since the start of the Coronavirus disease 2019 (COVID-19) outbreak. The epidemic stage of COVID-19 has brought great challenges to the diagnosis and management of colorectal cancer (CRC) patients. Symptoms, such as fever and cough caused by cancer, and the therapeutic process (including chemotherapy and surgery) should be differentiated from some COVID-19 related characteristics. Besides, clinical workers should not only consider the therapeutic strategy for cancer, but also emphasize COVID-19's prevention. Moreover, the detailed therapeutic regimens of CRC patients may be different from the usual. Also, treatment principles may various for CRC patients with or without severe acute respiratory syndrome coronavirus 2 (SARS-CoV-2) infection, as well as patients with or without an emergency presentation. In this paper, we want to discuss the above-mentioned problems based on previous guidelines, the current working status and our experiences, to provide a reference for medical personnel.
\end{abstract}

Keywords: Severe acute respiratory syndrome corona virus 2 (SARS-CoV-2); 2019 novel coronavirus disease (COVID-19); colorectal cancer (CRC)

Submitted Mar 03, 2020. Accepted for publication Mar 18, 2020.

doi: 10.21037/atm.2020.03.158

View this article at: http://dx.doi.org/10.21037/atm.2020.03.158

\section{Introduction}

Recently, the world-wide outbreak of newly emerged human coronavirus, known as the severe acute respiratory syndrome coronavirus 2 (SARS-CoV-2), has evolved as a threat to global health security (1-3). Coronavirus disease
2019 (COVID-19) caused by SARS-CoV-2 is mainly characterized by infected respiratory symptoms, including fever, dry cough, dyspnea and infiltrate on chest radiograph. By March 7, 2020, the total number of confirmed COVID-19 cases in China had reached 80,813, of which 3,073 died (data from the National Health Commission of 
China). Although positive responsive measures taken by the Chinese government resulting in a continuous and steady decrease in the figure for confirmed cases nationwide, the number of confirmed COVID-19 patients is still on the rise in other countries and regions. As of today, South Korea $(6,767)$, Iran $(4,747)$ and Italy $(4,636)$ hold the highest number of confirmed cases outside China.

Colorectal cancer (CRC) is a common malignant tumor of the digestive tract with age-standardized incidence rate of 1.752 per million people $(4,5)$. As a wasting disease, CRC itself and its corresponding treatment may weaken the immune response to respiratory bacteria, makes patients more susceptible to virus infection. A recent study showed that about $1 \%$ of SARS-CoV-2 infected patients had a history of cancer, which seemed to be higher than the overall incidence $(0.29 \%)$ of cancer in the Chinese population $(6,7)$. Besides, SARS-CoV-2 infected patients who accompanied with cancers may in turn make the COVID-19 worse. The case-fatality rate is reported higher in those with preexisting comorbid conditions. Patients with both COVID-19 infection and cancer have a 5.6\% case-fatality rate, which is more than 2 times higher than the overall case-fatality rate $(2.3 \%)(8,9)$. Furthermore, SARS-CoV-2 is proved to be detected in gastrointestinal tract and urine, these potential routes of transmission cannot be ignored especially in the diagnosis and treatment of digestive tract diseases. Therefore, in the context of the current COVID-19 outbreak, the management of CRC patients deserves attention.

As a designated hospital, our institute has gained in certain experience for COVID-19 treating and surgical management during the outbreak (10-12). Here, we discuss the diagnosis and management plan of patients with CRC during the COVID-19 outbreak based on previous guidelines, the current working status and our experiences, to provide a reference for clinical practitioners.

\section{Medical screening and diagnosis of the colorectal cancer}

All outpatients must firstly be taken their temperature and be carefully inquired about the epidemiological history, including (I) whether they have been to epidemic focus areas within 14 days, (II) whether there is a history of travel or residence in the community and surrounding areas of COVID-19 cases reported, (III) whether to contact people from epidemic areas especially Wuhan and the confirmed/ suspected infectors, and (IV) whether have respiratory symptoms such as fever, cough, dyspnea, or whether have the history of wild animal exposure. A chest imaging examination should be carried out for outpatients. Notably, we should beware of the potential false-negative results in temperature checking and imageological finding. A recent report showed that no chest imaging abnormality was found in $17.9 \%$ of patients with non-severe COVID-19 and $2.9 \%$ of patients with severe COVID-19. Likewise, only $43.8 \%$ of patients presented as a fever on admission (13). Hence, the virus detection is recommended to be the diagnostic gold standard. SARS-CoV-2 can be detected in the respiratory tract (nasopharyngeal, oropharyngeal, endotracheal aspirate, and bronchoalveolar lavage), the blood and the digestive tract (feces) (14). And the diagnostic criteria of SARS$\mathrm{CoV}-2$ infection are as follow: (I) specimens tested via realtime polymerase chain reaction (RT-PCR) shows positive for SARS-CoV-2 nucleic acid; (II) virus gene sequencing shows highly homologous to that of the known SARSCoV-2; or (III) serological identification shows SARS$\mathrm{CoV}-2$ antibody (IgM or IgG) positive (15-18).

After rule out the SARS-CoV-2 infection, patients will be transferred to the corresponding specialized clinic for further diagnosis and treatment. Non-invasive detections should be selected first for the screening of colorectal patients. In addition to the respiratory tract, recent research shows that virus strain may exist in the feces and digestive tract of SARS-CoV-2 infected patients (19). Hence, the physical examinations should be carried out with effectively protective measures, especially the digital rectal examination. Specialists can take systematic preoperative examinations to collect clinic, laboratory and radiological characteristics data for those patients who show gastrointestinal symptoms, have abdominal mass, or rectal neoplasm. The gold standard for the diagnosis of CRC is to take materials via electronic colonoscopy for pathological confirmation. Besides, endoscopy may also be an effective way to treat those patients who have gastrointestinal hemorrhage or ileus without peritonitis. However, the previous endoscopic disinfection standard may not guarantee the inactivation of new coronavirus, which may lead to the risk of doctor-patient and patientpatient cross infections. Therefore, for confirmed/ suspected patient with SARS-CoV-2 infection, endoscopic procedures should be performed in a special isolated room or negative pressure operation room (OR), the instruments and the endoscopic room should be sterilized strictly after the examination (by vaporized hydrogen peroxide sterilizer for at least 2 hours) (20). 


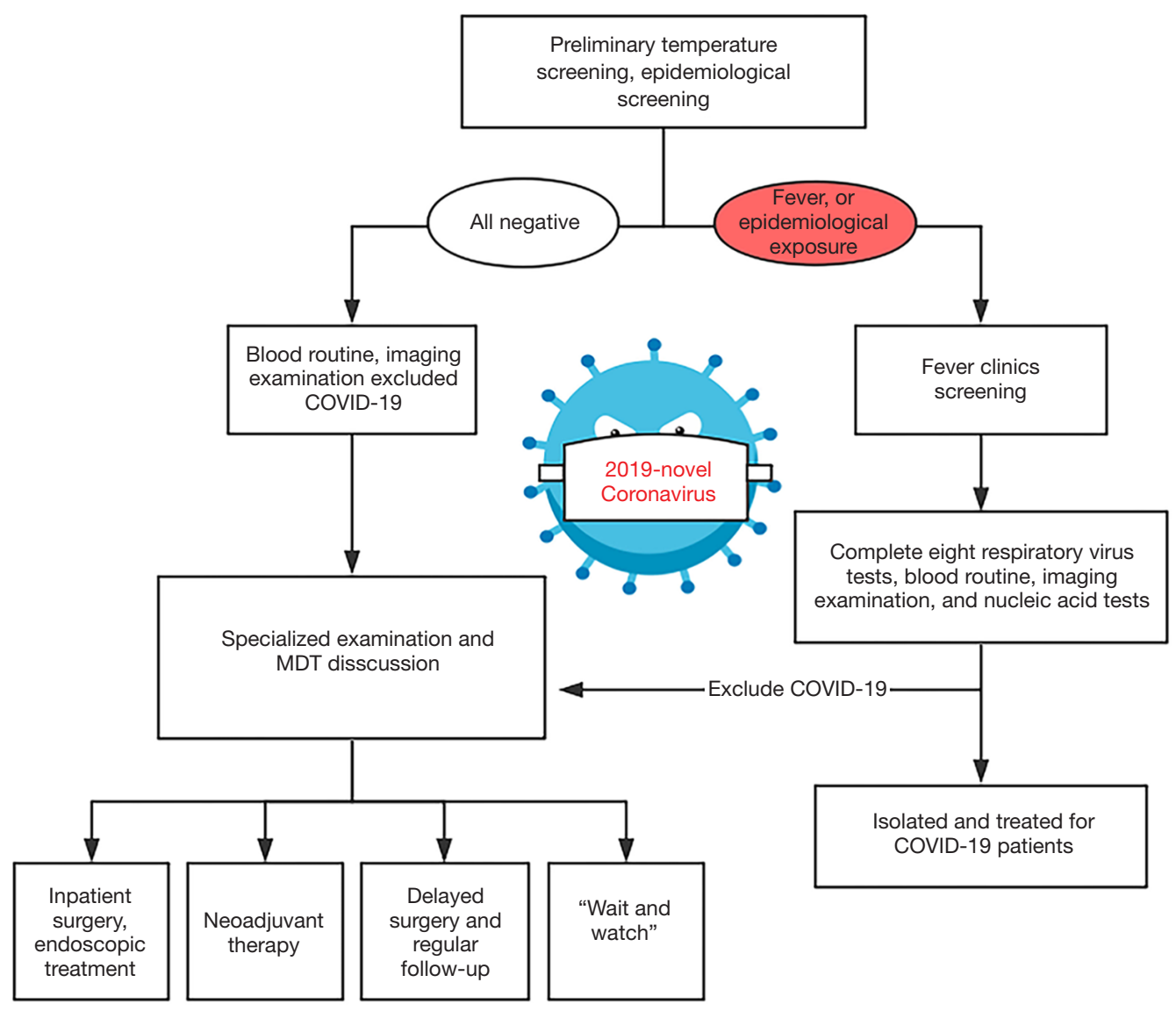

Figure 1 Diagnosis process of the colorectal cancer patients during the outbreak period of COVID-19 infection. COIVD-19, Coronavirus disease 2019; MDT, multidisciplinary team.

It should be noted that, some of the rare clinical symptoms of CRC need to be distinguished from the SARS-CoV-2 infection to avoid misdiagnosis. Lung is the second most easily metastatic site except liver for CRC, and compared to colon cancer, the carcinoma of rectum is more prone to develop lung metastasis (21). CRC Patients with lung metastases could have characteristics similar to COVID-19, such as cough, fever and even the pulmonary shadow. These deceptive symptoms should be identified by viral nucleic acid test, CT imaging, and tumor markers. Obvious multiple nodules of the lungs, pleural thickening, or pleural effusion may be the main imaging findings in CRC patients with lung metastases, while in patients with SARS-CoV-2 related pneumonia, the lung imaging often manifested as patchy shadow or ground glass opacity (12). Fever in CRC patients with intestinal obstruction may result from the intestinal bacteria entering into the blood stream. Besides, neutropenic and other side effects caused by chemotherapy, as well as cancer itself could lead to fever, which also needs to be discriminated from the fever caused by SARS-CoV-2 infection.

The specific flow chart of the admission process for CRC patients during the outbreak is shown in Figure 1. In brief, patients with clinically highly suspected or initially diagnosed CRC should undergo rigorous screening in the outpatient setting. After a detailed epidemiological history taking, SARS-CoV-2 infection screening and careful physical examination, CRC patients will be admitted for further diagnosis and treatment. Given this special period, the current multidisciplinary team (MDT) should be composed of respiratory medicine, infection, colorectal surgery, oncology, digestion, imaging, pathology departments and intensive care unit (ICU). And the MDT consultation is recommended to be arranged through Voice of Internet Phone (VOIP) and television screen meeting. The main tasks of the MDT are as follows: 
Table 1 Clinical classifications of SARS-CoV-2 infected cases based on the "Diagnosis and management plan of pneumonia with new coronavirus infection (trial version 7)" (17)

\begin{tabular}{ll}
\hline Clinical classifications & Description \\
\hline Asymptomatic infection & Viral nucleic acid test result positive but lacking typical symptoms including fever, dry cough, and fatigue \\
Mild infection & Non-pneumonia with mild clinical symptoms \\
Moderate infection & Pneumonia with fever and respiratory symptoms \\
Severe infection & (I) Dyspnea, respiratory frequency $\geq 30 / \mathrm{min} ;$ \\
& (II) Blood oxygen saturation $\leq 93 \%$; \\
(III) Partial pressure of arterial oxygen to fraction of inspired oxygen ratio $<300 \mathrm{mmHg}$; or \\
(IV) Lung infiltrates $>50 \%$ within 24 to 48 hours (any one of above) \\
(I) Respiratory failure; \\
(II) Septic shock; or \\
(III) Multiple organ dysfunction or failure (any one of above)
\end{tabular}

(I) Avoiding misdiagnosis and missed diagnosis of SARS-CoV-2 infection, avoiding cross infection;

(II) Completing the task of CRC's diagnosis collaboratively by utilizing the existing medical resources;

(III) Providing optimal individualized treatment, maximizing the clinical benefit of patients; and

(IV) Developing a well-designed follow-up strategy, providing online clinical consultation services.

\section{Treatment strategies for CRC during COVID-19 epidemic}

The treatments of CRC include interdisciplinary surgery, radiotherapy, chemotherapy, targeted therapy and even immunotherapy. Now comes an outbreak of COVID-19, although the principles of tumor therapy remain unchanged, the detailed therapeutic methods of CRC patients may be different from the usual.

A population-based study shows that the majority of patients $(53.6 \%)$ infected by SARS-CoV-2 at the age of 50 or older, which is, by and large, in coincidence with the predilection age of colorectal cancer $(22,23)$. On the other hand, in some colorectal cancer patients, the virus may "awaken" after the incubation period of SARS-CoV-2 infection. Besides, about $1 \%$ of SARS-CoV-2 infected cases are asymptomatic (viral nucleic acid test result positive but lacking typical symptoms such as fever, cough, and pneumonia), which complicated the illness judgment (Table 1). Aggressive treatment may further lower immunity, making CRC patients more susceptible to COVID-19. Therefore, the overall regimen for tumor treating should be appropriately simplified. Meanwhile, in addition to tumor therapy, prevention of the COVID-19 (for non-SARSCoV-2 infected patients) and promoting the rehabilitation of COVID-19 (for SARS-CoV-2 infected patients) should be two other treatment focuses.

In the following, we separately discuss the corresponding treatment strategies for CRC patients with or without SARS-CoV-2 infection, and patients with or without an emergency presentation.

\section{Management of non-emergency}

\section{Non-emergency cases with confirmed/suspected COVID-19}

Urgent respiratory and epidemiologic consultations are warranted for CRC patients with suspected or confirmed COVID-19. These patients should be directly transferred to the isolation ward. As mentioned above, the prognosis for those SARS-CoV-2 infected patients who have preexisting diseases is often worse than a simple infection of SARS$\mathrm{CoV}-2$. Besides, early asymptomatic or atypical state but rapid progression at later in some infected patients may bring difficulty to the prediction of COVID-19 progression. Wang et al. reported the data of 138 hospitalized patients with COVID-19, among them, 26\% required ICU care. Of 47 discharged patients, the mean time from onset to dyspnea was 5.0 days, and 8.0 days to develop acute respiratory distress syndrome (ARDS). Interestingly, more 


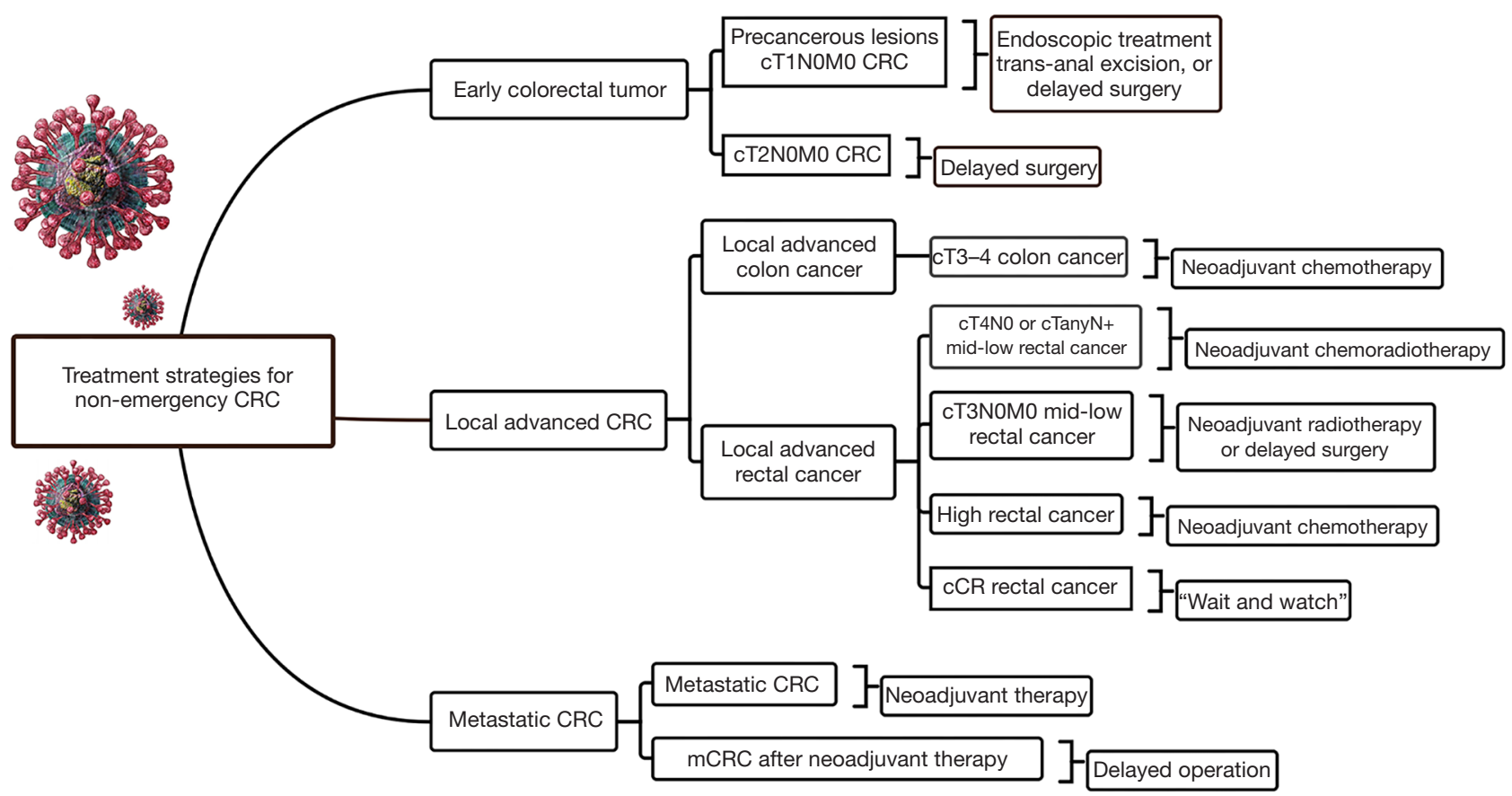

Figure 2 Recommended treatment strategies for non-emergency colorectal cancer cases without SARS-CoV-2 infection during the epidemic. CRC, colorectal cancer; cCR, clinical complete response; mCRC, metastatic colorectal cancer.

than one-tenth of hospitalized patients presented initially with atypical symptoms such as nausea and diarrhea (12). Furthermore, the incubation period from exposure to symptoms for most patients with COVID-19 varies from 3 to 14 days, suspected infected patients should be, therefore, quarantined for at least 14 days to ensure safety (24-26). Hence, for CRC patients with confirmed/suspected COVID-19 but without presenting a surgical emergency, the treatment should be more focused on SARS-CoV-2infection-related diseases. A short delay in CRC therapy may have no remarkable influence on the prognosis of the patients with CRC, if necessary, CRC therapy could be suspended (27).

\section{Non-emergency cases without COVID-19}

Simplifying treatment, providing psychological comfort, and preventing SARS-CoV-2 infection should be the main treatment principles for treating non-emergency CRC patients without novel coronavirus infection. The following recommendations are drafted according to CRC patients at different stages. The specific flow chart of treatment strategies is shown in Figure 2.

\section{Treatment strategies for patients with early stage CRC}

Tumor at an early stage often confined to the mucosa or submucosa, and most of them can be radically treated via endoscopy with satisfactory outcomes (28). Trans-anal excision is another suitable technique for patients with early rectal tumors whose tumor diameter less than $3 \mathrm{~cm}$ and within $8 \mathrm{~cm}$ from the anal margin (29). During this period, endoscopic resection techniques including endoscopic mucosal resection (EMR) and endoscopic submucosal dissection (ESD) or trans-anal excision could be used to be performed those patients who with precancerous lesion or early stage CRC (cT1N0M0). Lymph node metastasis is one of the important factors affecting the prognosis of CRC. Adequate imaging evaluation is mandatory before trans-anal excision and endoscopic treatment.

The radical surgery is the major therapy for patients with CRC staged by cT2N0M0. In the epidemic of COVID-19, however, surgery is recommended to be suspended especially in areas with limited protection conditions or severe epidemic situations. As mentioned previously, a brief delay in surgery may not exacerbate the disease.

Treatment strategies for patients with local advanced CRC Neoadjuvant chemotherapy (NACT) can not only shrink the size of the tumor, increase the rate of R0 resection, but potentially also eradicate some small satellite lesions (30,31). Data from the National Cancer Database (NCDB) 
showed that NACT could bring significant survival benefits to patients with T4b colon cancer (32). Similarly, NACT also shows satisfactory outcomes for treating patients with $\mathrm{T} 3$ or $\mathrm{T} 4 \mathrm{a}$ colon cancer. Liu et al from China reported 47 local advanced colon cancer patients (T3/T4a-b) treated by CapOX (oxaliplatin plus capecitabine) regimen, the authors concluded that NACT with CapOX was an effective and safe option for these patients (33).The 2019 American Society of Clinical Oncology (ASCO) conference also reported the survival results of the FoxTROT study, which showed the excellent effect of the FOLFOX regimen (34). During the epidemic period, therefore, as an effective "bridge" therapy, NACT with CapOX or FOLFOX regimen could be recommended for treating patients with cT3-4b stage of colon cancer. Besides, the Chinese Society of Clinical Oncology (CSCO) guideline recommends that mid-low rectal cancer with cT3-4N0 or cTanyN+ can be treated with concurrent chemoradiotherapy before radical resection (35). Similarly, in this special period, neoadjuvant chemoradiotherapy is strongly recommended for local advanced mid-low rectal cancer. In brief, radiotherapy (45-50.4 Gy/25-28 F) and NACT (capecitabine or mFOLFOX6) could be chosen for mid-low advanced rectal cancer patients with T4 stage. And for rectal cancer patients with T3 stage, short-term radiotherapy with lower radiation dose (25 Gy/5 F) may be an option.

\section{The optimal timing for surgery and the "Wait and watch $(W \& W)$ " strategy for rectal cancer during COVID-19 epidemic}

Although total mesorectal excision (TME) has been used for a long time as a cornerstone for rectal cancer treatment, the concept of "W\&W" has gained popularity in recent years, representing a divergence from the traditional treatment (36). Previous study has proved that about $15-30 \%$ of patients with rectal cancer treated with neoadjuvant treatment develop pathologic complete response (pCR) (37). Clinical complete response (cCR) may also be common in those low rectal cancer patients who finish neoadjuvant chemoradiotherapy. A recent research from the International Watch \& Wait Database (IWWD) reported 1,009 cases who underwent "W\&W" strategy after neoadjuvant treatment between 2015 and 2017. The 5 -year overall and disease-free survival were $85 \%$ and $94 \%$, respectively, and cCR was found in $880(80.7 \%)$ patients (38). Another report showed that although 89 out of 385 patients developed a local regrowth after a median of 9 months, most of them (94\%) could still undergo surgical treatment with relatively good prognosis (2-year overall and disease- free survival were $90.3 \%$, and $98.4 \%$, respectively) (39). The consensus of Chinese experts on the " $W \& W$ " policy (2020 version) also recommended that rectal cancer patients who achieve cCR or near-cCR after neoadjuvant treatment could enroll in the W\&W strategy (40). Therefore, during the outbreak, $W \& W$ strategies may be more suitable for rectal cancer patients, especially the elderly. And we recommend longer waits between follow-up visits to reduce the nosocomial exposure time. In addition to traditional tumor markers, circulating tumor cell (CTC) and circulating tumor DNA (ctDNA) monitoring may provide information regarding tumor status and may therefore be complementary to MRI and endoscopic ultrasonography, these testing technologies are worthy of spreading in hospitals where the conditions are permitted (41-43). Several factors (including the prolonged effect of neoadjuvant therapy, the physical and nutritional status of patients and the risk of tumor progression) may determine the timing of resection for rectal cancer after neoadjuvant treatment. A longer time to surgery may potentially allow the tumor to continue regressing. However, the risk of primary or metastatic tumor regrowth is also increasing. The optimal timing for tumor response assessment remains a matter of debate. The NCCN Clinical Practice Guidelines recommended that assessing for tumor response at week 5 to 12 from the end of neoadjuvant treatment (44). In the epidemic of COVID-19, the timing for the operation could be appropriately delayed, which may be extended to 16-24 weeks after neoadjuvant treatment (45). Additional 2-4 cycles of CapeOX may be scheduled for selected rectal cancer patients to increase the odds of pCR when waiting for the surgery (46).

\section{Treatment strategies for patients with metastatic colorectal cancer $(m C R C)$}

The liver is the most common CRC metastatic site, accounting for about $15-25 \%$ of CRC patients (47). During the epidemic period, initially diagnosed mCRC patients without presenting a surgical emergency could be recommended to receive neoadjuvant therapy (such as CapeOX regimen and targeted therapy) (48). After neoadjuvant therapy, MDT is responsible for deciding the timing of surgery for patients with mCRC and the surgery could be appropriately delayed. Since patients with mCRC always have larger tumor burdens and in relatively worse physical condition, the one-stage surgery of primary and metastatic resections is not recommended by some researches during the epidemic period of COVID-19 (48).

To sum up, during the COVID-19 outbreak, all CRC 
cases without SARS-CoV-2 infection should undergo a regular follow-up. Further positioning detections such as a contrast enhanced CT or MRI will be conducted when clinically indicated or when tumor progression was suspected. Besides, the chemoradiotherapy related adverse reactions should not be ignored. However, reducing exposure time is an important element in preventing SARS-CoV-2 infection. Therefore, it is recommended that patients take oral chemotherapy instead of intravenous chemotherapy and patients choose the nearest hospital to receive necessary treatment. And the interval of NACT could be extended appropriately.

Medical institutions should provide basic services including answering medical questions, instructing medication treatments and providing psychological guidance to CRC patients. Fortunately, most of these services can be successfully carried out on the internet. After the end of the outbreak, the treatment plan should be re-developed based on each patient's response to previous treatment and conditions.

\section{Management of emergency patient}

Although most CRC surgeries are confine operations with adequate preparations, a significant number of patients still require immediate intervention for emergency presentations such as obstruction, perforation or severe bleeding (49). Recent studies showed that approximately $1 / 4-1 / 3$ of CRC cases underwent emergency surgery (50-52). As previously mentioned, CRC patients presenting as an emergency tend to be older, have lower immunities and more advanced tumors, resulting in a prolonged recovery time and a relatively poor prognosis (51). Aggravative treatment may not carry a beneficial effect on the prognosis of these patients (53). Besides, during the epidemic period of COVID-19, medical resources are relatively scarce especially in the areas badly affected by the outbreak (such as Wuhan, Hubei province). Fewer blood donors have also led to a shortage of plasma. Hence, CRC patients who present as emergencies require more attention in the epidemic of COVID-19.

\section{Emergency cases without COVID-19}

Minimizing trauma to patients, reducing hospital stays, and preventing SARS-CoV-2 infection should be the main treatment principles for treating emergency cases without SARS-CoV-2 infection. Most CRC with obstruction, perforation or hemorrhage is late-stage patients with relatively low R0 resection rates in emergency surgery (54).

Before surgery, all patients will undergo respiratory specimens and blood samples detection to rule out SARSCoV-2 infection. For patients with bowel obstruction in relatively good physical condition after the failure of the conservative therapy, the endoscopic colonic stenting procedure using self-expanding metallic stents (SEMS) may be considered first (55). About $70-80 \%$ of malignant bowel obstruction is located in the left-sided colon, which makes them more feasible for endoscopic intervention (56). If successful, endoscopic colonic stenting can not only effectively alleviate obstruction, but also help CRC patients to get through the outbreak period. Continuing or starting NACT may further shrink the size of tumors even to achieve downstaging. In short, endoscopic colonic stenting acts as a bridge to curative surgery, represents an alternative to colostomy, and further reduces the risk of SARS-CoV-2 infection for patients with bowel obstruction, but we should pay attention to the incidence of colonic perforation during endoscopy for patients with ileus (57). However, stenting is not recommended for patients with low rectal cancer because it may cause fecal incontinence, urgency and tenesmus (58).

Fecal occult blood is the most common sign whereas massive bleeding is relatively rare in hemorrhagic CRC patients. The treatment plan remains overall unchanged, including endoscopic treatment, angiographic treatment, and surgical options. Fortunately, over $70 \%$ of acute hemorrhagic CRC patients could be cured by conservative treatment only (59). Conservative and endoscopy treatment may be used as first-line therapies for treating moderate and even massive bleeding when facing the epidemic of COVID-19.

Emergent operation is still reserved for CRC patients with refractory bowel obstruction, massive hemorrhage or perforation despite other interventions of cessation. For these patients without SARS-CoV-2 infection, resolving the emergency event and simultaneously radical resection should be considered when possible. If there are no obvious contraindications, laparoscopic surgery may be recommended for patients to lessen surgical trauma. Enhanced recovery after surgery (ERAS) is particularly recommended for postoperative CRC patients to reduce the postoperative complications, shorten hospital stay, and more importantly, minimize the risk of nosocomial infection.

Emergency cases with confirmed/suspected COVID-19 To date, agreed guidelines on how to deal with CRC patients with confirmed or suspected COVID-19 who 
Table 2 Recommended three-grade occupational protection strategies for clinicians during the outbreak period of COVID-19 infection

\begin{tabular}{|c|c|c|c|c|c|c|c|c|c|c|}
\hline $\begin{array}{l}\text { Protection } \\
\text { level }\end{array}$ & Environment & \multicolumn{9}{|c|}{ Protective Gear } \\
\hline Grade 1 & $\begin{array}{l}\text { Triage, surgical clinic, } \\
\text { general ward of surgery }\end{array}$ & + & + & + & + & + & + & + & - & - \\
\hline Grade 2 & $\begin{array}{l}\text { Isolation ward, Medical } \\
\text { staff who transfer } \\
\text { suspected or confirmed } \\
\text { patients }\end{array}$ & + & + & + & - & + & + & + & + & - \\
\hline Grade $3^{\dagger}$ & $\begin{array}{l}\text { Surgeon, anaesthetist, } \\
\text { instrument nurse }\end{array}$ & + & + & + & - & + & + & + & + & + \\
\hline
\end{tabular}

${ }^{\dagger}$, grade 3 protection is recommended to be adopted for medical staff when performing surgical intervention for confirmed/suspected SARS-CoV-2 infected patients or patient without virus detection. COIVD-19, Coronavirus disease 2019; SARS-CoV-2, severe acute respiratory syndrome coronavirus 2 .

presenting as emergencies remain scarce and incomplete. For those patients with incomplete intestinal obstruction or mild to moderate bleeding, non-surgical strategies including conservative treatment, endoscopic techniques and endovascular embolization could be chosen firstly to lower stoma rate. Although the emergent operation may be an extremely risky practice in patients with concurrent severe SARS-CoV-2 infection, it may still be the only life-saving procedure for those who developed uncontrolled massive bleeding and acute diffuse peritonitis caused by intestinal perforation. Undoubtedly, treating these patients brings great challenges to MDT, especially ICU, department of anesthesiology and colorectal surgeons.

Emergency CRC surgical treatment is recommended to be performed in the designated hospital and operated in negative pressure $(-5$ pa) OR. All treating team members should use enhanced grade- 3 occupational protection (the highest level) including wearing medical cups, N95 or higher-level respirators, medical coverall, eye shield, surgical latex gloves, disposable operating coat, protective hood, waterproof shoe covers, and disposable shoe covers (Table 2, Figure 3). Anesthesiologists should wear an additional disposable medical face shield to prevent patients' coughed droplets during trachea intubation and extubation.

Although transmission by air-borne droplets and contaminated hands are considered the major routes of SARS-CoV-2 spreading, the aerosol may be another contributing factor (17). Surgeons should dissect meticulously to avoid splashing of body fluid during the operation. Energy based surgical instruments (such as high-frequency electrotomes and ultrasonic knives) related smoke mixed with the blood and exudate of the patient could form into aerosol and diffuse into the surrounding air (60). Although effective at removing exudate and blood, the surgical aspirator dose not adequate for filtering surgical aerosol. As a possible solution, additional air purifiers may be placed in OR. Likewise, the current consensus is that laparoscopic surgery without smoke evacuation filters is not recommended to SARS-CoV-2 infected patients because it is unclear whether laparoscopic surgery produces a higher density of aerosols which may increase the risk of infecting healthcare workers in case the air leakage from pneumoperitoneum (61).

Radical surgery should be chosen cautiously after a multidisciplinary assessment. Most specialists recommend not to perform complex anastomosis. And the indication for enterostomy should be broadened properly (62). In emergency CRC patients with relatively severe organ damage, aggressive radical resection could further compromise the patient's immune system. If this is the case, ostomy may be a good option. Besides, recent researches demonstrating that both severe acute respiratory syndrome coronavirus (SARS-CoV) and SARS-CoV-2 share the same host cell receptor [angiotensin-converting enzyme 2 (ACE2)], and the latter shows 10-20-fold higher affinity binding to ACE2 that the SARS-CoV $(63,64)$. Although the amount of ACE2 protein in the colon tissue remains controversial $(61,63)$, ACE2 expression and SARS-CoV replication are quite common in all parts of the small intestine which could also be the potentially infected site of SARS-CoV-2 (65-67). Extrapulmonary manifestations such as watery diarrhea and vomiting in patients with 


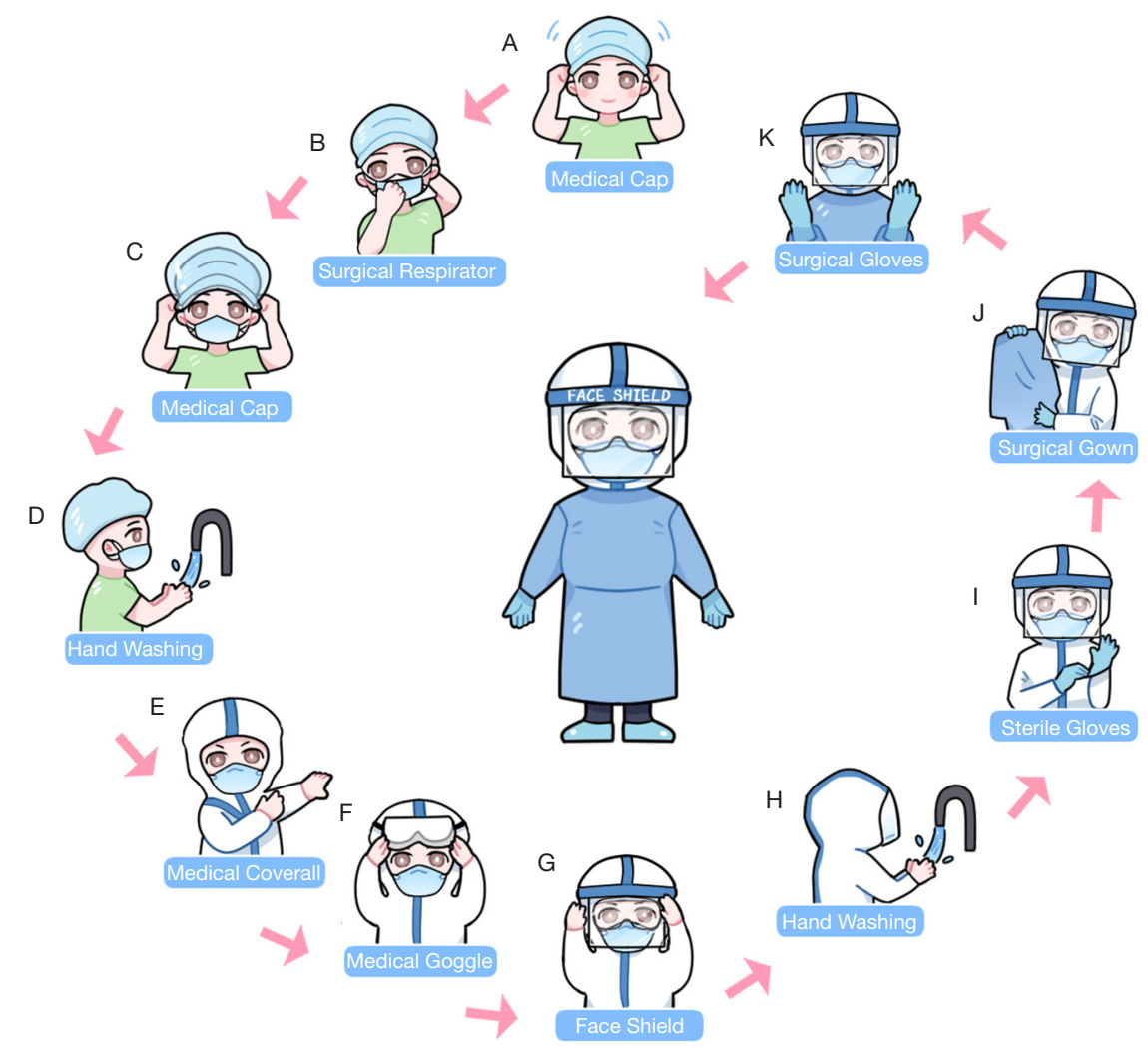

Figure 3 Recommended personal protective measures for medical staff when performing a surgical intervention for suspected/confirmed COVID-19 patients. Adapted from reference (18) with permission.

SARS-CoV-2 infection have been reported $(10,11,68,69)$. Data from our hospital showed that more than $1 / 3$ of the COVID-19 infected patients $(55 / 139,39.6 \%)$ complained about gastrointestinal symptoms, among them 18 cases (12.9\%) had diarrhea (12). Resection with fecal diversion may reduce the incidence of anastomotic complications related to gastrointestinal symptoms induced by SARS$\mathrm{CoV}-2$ infection.

After the operation, the OR should be thoroughly disinfected and is recommend to be closed for at least 2 hours. All patients should be treated with close monitoring of vital signs (monitored emphatically), oxygen therapy, antivirals and allowance of nutrients. Chest CT will be reexamined at the suggestion of the respiratory/infection department. It is worth noting that researchers from multiple organizations have successfully isolated novel coronavirus strains from stool specimens of infected patients (13). Therefore, additional attention should be paid to the risk of fecal-oral transmission during postoperative stoma care. Notably, the results of nucleic acid detection may be "deceptive", which seems to carry the risk of "weakening" medical staffs' psychological vigilance and protection measures (70).

\section{Differential diagnosis between COVID-19 related fever and postoperative fever}

Approximately half of SARS-CoV-2 infected patients had a fever at the onset of illness, but developed in about $90 \%$ after hospitalization (10-13,69). Similarly, fever is also one of the most common complications after surgery, with a vary widely incidence rate (range from $12 \%$ to $90 \%)(71-73)$. The real causes of postoperative fever need to be accurate judged by clinicians. Besides, febrile nonhemolytic transfusion reactions may bring difficulty to the identification of causes of postoperative fever. Therefore, unnecessary transfusions are not recommended during the COVID-19 outbreak. Here, we discuss the distinction between three common postoperative fevers and COVID-19 related fever (Table 3). 
Table 3 Characteristics of the main cusses of fever postoperatively during the outbreak period of coronavirus disease 2019 (COVID-19) infection

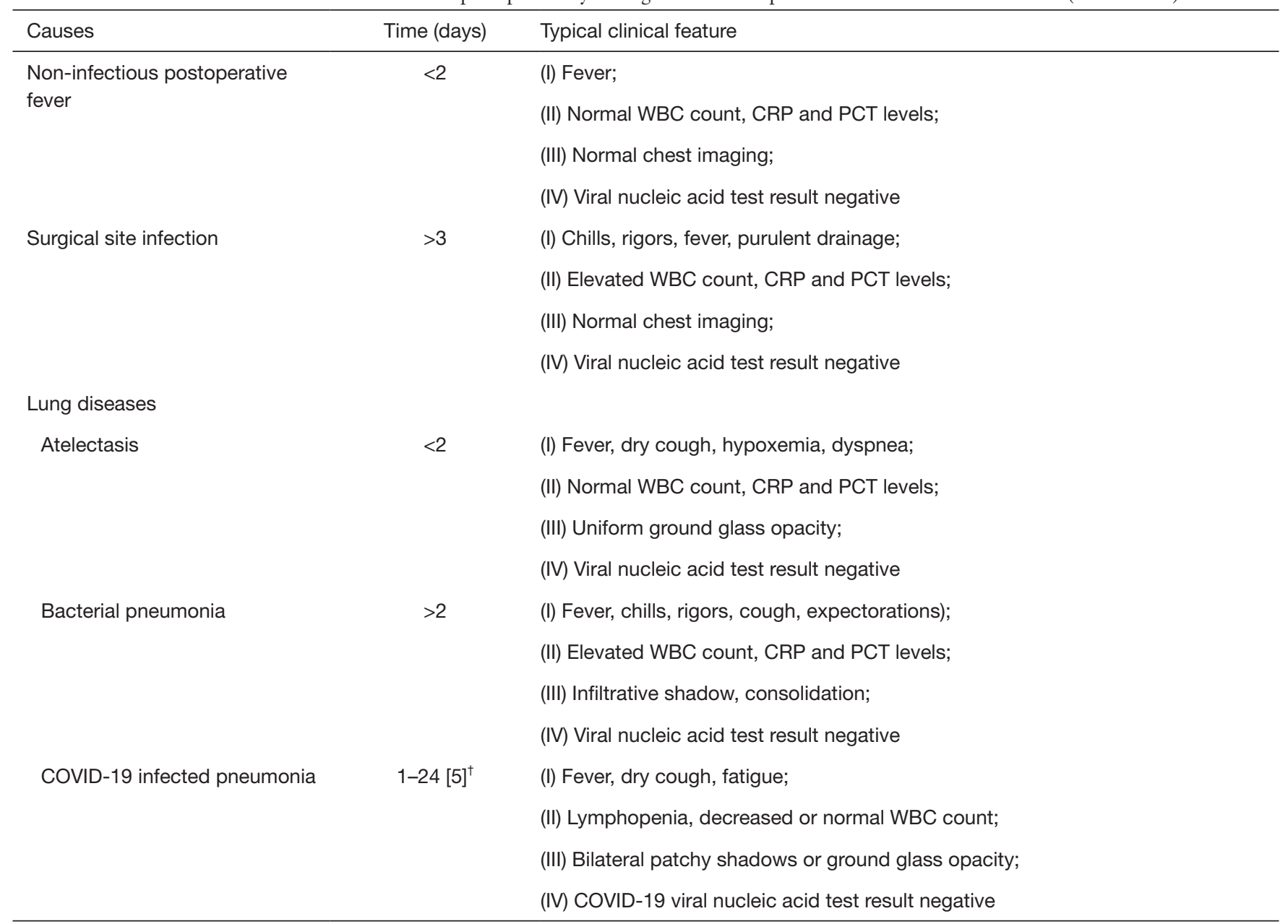

${ }^{\dagger}$, the number is recorded as incubation period. COVID-19, coronavirus disease 2019; CRP, C-reactive protein; PCT, procalcitonin; WBC, white blood cell.

\section{Noninfectious postoperative fever}

More than half of postoperative fever cases occur within the first 48 hours. Among them, noninfectious fever accounts for the majority. Inflammatory response and cellular injury caused by surgical stress have been regarded as major culprits leading to early postoperative fever (74). The median incubation period of COVID-19 from exposure to symptoms is about 4 days (interquartile range, 2 to 7) (13), which is diverse from noninfectious postoperative fever. Besides, COVID-19 mainly attacks the respiratory system, coughing is another common symptom of SARS-CoV-2 infection which is uncommon in noninfectious postoperative fever. Serological testing, chest radiography or CT scan might help to differentiate noninfectious postoperative fever from COVID-19.
SARS-CoV-2 RNA can also be detected in respiratory specimens by RT-PCR assay in a relatively short time.

\section{Surgical site infection}

Surgical site infection can be broadly separated into three parts: superficial, deep (including muscle and fascia), and organ/space infection. The clinical manifestations of surgical site infection include chills, rigors, fever, swelling, peritonitis with or without purulent drainage and most patients develop symptoms 5 to 10 days after surgery (75). Although surgical site infection and COVID-19 may share similar median time to onset. The most common pathogens that cause surgical site infection are Staphylococcus aureus, follow by Gram-negative bacilli 
coagulase-negative staphylococci, and Streptococci (76). Therefore, surgical site infection happens with significant increases in white blood cell (WBC) count, C-reactive protein (CRP) and procalcitonin (PCT) levels, which are contrary to COVID-19. Besides, abdominal and pelvic CTs may be helpful to identify deep or organ/ space infections. The differential diagnosis may be straightforward in the presence of classic symptoms. It should be noted that, however, the two diseases may coexist in the same patient. One should not be fooled by surgical site infection with obvious symptoms in ignoring the possibility of COVID-19.

\section{Atelectasis and non-SARS-CoV-2 infected pneumonia}

Atelectasis, mainly found in postoperative bedridden persons, is another common complication in the early postoperative period (within 48 hours). Postoperative CRC patients in immunosuppressed states are susceptible to develop infectious pulmonary diseases including bacterial and non-bacterial pneumonias. Besides, atelectasis could become the starting point of a wide range of other pneumonia over time. Symptoms and signs of atelectasis and non-SARS-CoV-2 infected pneumonia both include fever, cough, hypoxemia, decreased breath sounds, dyspnea and abnormal findings on chest radiography, which may make it difficult to differentiate non- SARS-CoV-2 infected diseases from COVID-19 based on symptoms and imaging. However, other systemic symptoms in nonSARS-CoV-2 infected pneumonia such as chills, rigors and expectorations may be less common in COVID-19. Besides, Postoperative bacterial pneumonias often lead to increased WBC count, CRP and PCT levels which may help establish a differential diagnosis. However, in both SARS-CoV-2 infected pneumonia and other non-bacterial pneumonias, the total WBC counts may be decreased or normal. If pneumonia is suspected, respiratory specimens and/or blood samples should be obtained (from throat swab or bronchoalveolar lavage) for microbiological diagnosis (such as gram stain and culture) and RT-PCR assay (or viral gene sequencing).

As mentioned above, during potentially coincident COVID-19 and non-COVID-19 infections, the possibility that a patient may contract both cannot be ruled out. In addition to routinely examining COVID-19, we also recommend testing for other pathogenic microorganisms such as influenza A and B.

\section{Conclusions}

In conclusion, we have tried to discuss the management plan of patients with CRC during the current outbreak of novel coronavirus infection. Although studies for COVID-19 caused by SARS-CoV-2 infection have sprung up since the outbreak, much remains unknown and many questions remain to be answered about COVID-19. We sincerely hope to develop a detailed clinical guideline for the diagnosis and treatment of CRC patients during the epidemic outbreak stage of COVID-19.

\section{Acknowledgments}

Funding: This study was supported by a grant from Project of Improving the Ability of Diagnosis and Treatment of Difficult Diseases in Zhongnan Hospital of Wuhan University (direction of neoplastic disease).

\section{Footnote}

Conflicts of Interest: All authors have completed the ICMJE uniform disclosure form (available at http://dx.doi. org/10.21037/atm.2020.03.158). The authors have no conflicts of interest to declare.

Ethical Statement: The authors are accountable for all aspects of the work in ensuring that questions related to the accuracy or integrity of any part of the work are appropriately investigated and resolved.

Open Access Statement: This is an Open Access article distributed in accordance with the Creative Commons Attribution-NonCommercial-NoDerivs 4.0 International License (CC BY-NC-ND 4.0), which permits the noncommercial replication and distribution of the article with the strict proviso that no changes or edits are made and the original work is properly cited (including links to both the formal publication through the relevant DOI and the license). See: https://creativecommons.org/licenses/by-nc-nd/4.0/.

\section{References}

1. Yang S, Cao P, Du P, et al. Early estimation of the case fatality rate of COVID-19 in mainland China: a datadriven analysis. Ann Transl Med 2020;8:128.

2. Lu R. Genomic characterisation and epidemiology of 
2019 novel coronavirus: implications for virus origins and receptor binding. Lancet 2020;395:565-74.

3. Munster VJ, Koopmans M, van Doremalen N, et al. A Novel Coronavirus Emerging in China-Key Questions for Impact Assessment. N Engl J Med 2020;382:692-4.

4. Chen H, Li N, Ren J, et al. Participation and yield of a population-based colorectal cancer screening programme in China. Gut 2019;68:1450-7.

5. Chen W, He J, Sun K, et al. Cancer incidence and mortality in China, 2014. Chin J Cancer Res 2018;30:1-12.

6. Liang W, Guan W, Chen R, et al. Cancer patients in SARS-CoV-2 infection: a nation-wide analysis in China. Lancet Oncol 2020;21:335-7.

7. Zheng RS, Sun K, Zhang S, et al. Report of cancer epidemiology in China, 2015. Zhonghua Zhong Liu Za Zhi 2019;41:19-28.

8. Wu Z, McGoogan JM. Characteristics of and important lessons from the coronavirus disease 2019 (COVID-19) outbreak in China: summary of a report of 72314 cases from the Chinese center for disease control and prevention. JAMA 2020. [Epub ahead of print].

9. Epidemiology Working Group for NCIP Epidemic Response, Chinese Center for Dis-ease Control and Prevention, Chinese Center for Diseases Control and Prevention. The epidemiological characteristics of an outbreak of 2019 novel coronavirus diseases (COVID-19) in China. Zhonghua Liu Xing Bing Xue Za Zhi 2020;41:145-51.

10. Chen H, Guo J, Wang C, el al. Clinical characteristics and intrauterine vertical trans-mission potential of COVID-19 infection in nine pregnant women: a retrospective review of medical records. Lancet 2020;395:809-15.

11. Wang D, Hu B, Hu C, et al. Clinical Characteristics of 138 Hospitalized Patients With 2019 Novel CoronavirusInfected Pneumonia in Wuhan, China. JAMA 2020. [Epub ahead of print].

12. Zhang J, Dong X, Cao Y, et al. Clinical characteristics of 140 patients infected with SARS-CoV-2 in Wuhan, China. Allergy 2020 [Epub ahead of print].

13. Guan WJ, Ni Z, Hu Y, et al. Clinical Characteristics of Coronavirus Disease 2019 in China. N Engl J Med 2020. [Epub ahead of print].

14. Wang L, Shi Y, Xiao T, et al. Chinese expert consensus on the perinatal and neonatal management for the prevention and control of the 2019 novel coronavirus infection (First edition). Ann Transl Med 2020;8:47.

15. $\mathrm{Xu} \mathrm{W,} \mathrm{Li} \mathrm{J,} \mathrm{He} \mathrm{X,} \mathrm{et} \mathrm{al.} \mathrm{The} \mathrm{diagnostic} \mathrm{value} \mathrm{of} \mathrm{joint}$ detection of serum IgM and IgG antibodies to 2019-
$\mathrm{nCoV}$ in 2019-nCoV infection. Chin J Lab Med 2020 (in Chinese).

16. Guo H, Chen X, Su C, et al. Challenges and countermeasures of thoracic oncology in the epidemic of COVID-19. Transl Lung Cancer Res 2020. [Epub ahead of print].

17. General office of national health commission, Office of the state administration of traditional Chinese medicine. Diagnosis and management plan of pneumonia with new coronavirus infection (trial version 7). Available online: http://www.nhc.gov.cn

18. Zhang $\mathrm{W}$, Jiang $\mathrm{X}$. Measures and suggestions for the prevention and control of the novel coronavirus in dental institutions. Front Oral Maxillofac Med 2020;2:4.

19. Zhang H, Kang Z, Gong H, et al. The digestive system is a potential route of 2019-nCov infection: a bioinformatics analysis based on single-cell transcriptomes. bioRxiv 2020 [Preprint].

20. Shi Q, Wang P, Hu H, et al. Diagnosis and treatment of emergency digestive endoscopy in Zhongshan hospital of Fudan university during the outbreak of COVID-19. Chin J Dig Endosc 2020. [Epub ahead of print].

21. Mitry E, Guiu B, Cosconea S, et al. Epidemiology, management and prognosis of colorectal cancer with lung metastases: a 30-year population-based study. Gut 2010;59:1383-8.

22. Zhao F, Wang J, Yu H, et al. Neoadjuvant radiotherapy improves overall survival for $\mathrm{T} 3 / 4 \mathrm{~N}+\mathrm{M} 0$ rectal cancer patients: a population-based study of 20300 patients. Radiat Oncol 2020;15:49.

23. Chen $\mathrm{W}$, Zheng R, Baade PD, et al. Cancer statistics in China, 2015. CA Cancer J Clin 2016;66:115-32.

24. Fang F, Luo X. Facing the pandemic of 2019 novel coronavirus infections: the pediatric perspectives. Zhonghua Er Ke Za Zhi 2020;58:81-5.

25. Zhang N, Wang L, Deng X, et al. Recent advances in the detection of respiratory virus infection in humans. J Med Virol 2020;92:408-17.

26. Sahu KK, Mishra AK, Lal A. Comprehensive update on current outbreak of novel coronavirus infection (2019nCoV). Ann Transl Med 2020. [Epub ahead of print].

27. Huang C, Wang Y, Li X, et al. Clinical features of patients infected with 2019 novel coronavirus in Wuhan, China. Lancet 2020;395:497-506.

28. Hashiguchi Y, Muro K, Saito Y, et al. Japanese Society for Cancer of the Colon and Rectum (JSCCR) guidelines 2019 for the treatment of colorectal cancer. Int J Clin Oncol 2020;25:1-42.

29. Hakiman H, Pendola M, Fleshman J. Replacing Transanal 
Excision with Transanal Endoscopic Microsurgery and/ or Transanal Minimally Invasive Surgery for Early Rectal Cancer. Clin Colon Rectal Surg 2015;28:38-42.

30. Ren X, Li H, Diao M, et al. Impact of microscopically margin-positive resection on survival in children with hepatoblastoma after hepatectomy: a retrospective cohort study. Int J Clin Oncol 2020;25:765-73.

31. Ren X, Li H, Diao M, et al. Results of Surgical Resections With Positive Margins for Children With Hepatoblastoma: Case Series From a Single Asian Center. Pediatr Blood Cancer 2019;66:e27479.

32. Dehal A, Graff-Baker AN, Vuong B, et al. Neoadjuvant Chemotherapy Improves Survival in Patients with Clinical T4b Colon Cancer. J Gastrointest Surg 2018;22:242-9.

33. Liu F, Yang L, Wu Y, et al. CapOX as neoadjuvant chemotherapy for locally advanced operable colon cancer patients: a prospective single-arm phase II trial. Chin J Cancer Res 2016;28:589-97.

34. Seymour MT, et al. FOxTROT: An international randomized controlled trial in 1,052 patients evaluating neoadjuvant chemotherapy for colon cancer. 2019 ASCO Annual Meeting. Abstract 3504. Presented June 1, 2019.

35. Diagnosis And Treatment Guidelines For Colorectal Cancer Working Group CS. Chinese Society of Clinical Oncology (CSCO) diagnosis and treatment guidelines for colorectal cancer 2018 (English version). Chin J Cancer Res 2019;31:117-34.

36. Nacion AJD, Park YY, Yang SY, et al. Critical and Challenging Issues in the Surgical Management of LowLying Rectal Cancer. Yonsei Med J 2018;59:703-716.

37. Chow O, Garcia-Aguilar J. Maximizing neoadjuvant treatment response and watch and wait. In: Chang G, editor. Rectal cancer: modern approaches to treatment. Cham: Springer, 2018:277-93.

38. van der Valk MJM, Hilling DE, Bastiaannet E, et al. Long-term outcomes of clinical complete responders after neoadjuvant treatment for rectal cancer in the International Watch \& Wait Database (IWWD): an international multicentre registry study. Lancet 2018;391:2537-45.

39. van der Sande ME, Figueiredo N, Beets GL. Management and Outcome of Local Re-growths in a Watch-and-wait Prospective Cohort for Complete Responses in Rectal Cancer. Ann Surg 2020. [Epub ahead of print].

40. Chinese Watch \& Wait Database Research Cooperation Group(CWWD); Chinese Association of Surgeons, Chinese Society of Coloproctology, Chinese Medical Doctor Association; Chinese Society of Colorectal Surgery, Chinese Medical Association; et al. Consensus on the Watch and Wait policy in rectal cancer patients after neoadjuvant treatment (2020 version). Zhonghua Wei Chang Wai Ke Za Zhi 2020;23:1-9.

41. Osumi H, Shinozaki E, Yamaguchi K, et al. Early change in circulating tumor DNA as a potential predictor of response to chemotherapy in patients with metastatic colorectal cancer. Sci Rep 2019;9:17358.

42. Khakoo S, Carter PD, Brown G, et al. MRI Tumor Regression Grade and Circulating Tumor DNA as Complementary Tools to Assess Response and Guide Therapy Adaptation in Rectal Cancer. Clin Cancer Res 2020;26:183-92.

43. Troncarelli Flores BC, Souza E Silva V, Ali Abdallah E, et al. Molecular and Kinetic Analyses of Circulating Tumor Cells as Predictive Markers of Treatment Response in Locally Advanced Rectal Cancer Patients. Cells 2019;8:641.

44. Benson AB, Venook AP, Al-Hawary MM, et al. Rectal Cancer, Version 2.2018NCCN Clinical Practice Guidelines in Oncology. J Natl Compr Canc Netw 2018;16:874-901.

45. Habr-Gama A, Sabbaga J, Gama-Rodrigues J, et al. Watch and wait approach following extended neoadjuvant chemoradiation for distal rectal cancer: are we getting closer to anal cancer management? Dis Colon Rectum 2013;56:1109-17.

46. Tey J, Leong CN, Cheong WK, et al. A phase II trial of preoperative concurrent chemotherapy and dose escalated intensity modulated radiotherapy (IMRT) for locally advanced rectal cancer. J Cancer 2017;8:3114-21.

47. Valderrama-Treviño AI, Baltazar BM, Ceballos-Villalva JC, et al. Hepatic Metastasis from Colorectal Cancer. Euroasian J Hepatogastroenterol 2017;7:166-75.

48. Chang W, Feng Q, Zhu D, et al. Whole-course management strategy for colorectal cancer patients during COVID-19 epidemic. Chin J Digest Surg 2020;19:E004.

49. Lavanchy JL, Vaisnora L, Haltmeier T, et al. Oncologic long-term outcomes of emergency versus elective resection for colorectal cancer. Int J Colorectal Dis 2019;34:2091-9.

50. Baer C, Menon R, Bastawrous S, et al. Emergency presentations of colorectal cancer. Surg Clin N Am 2017;97:529-45.

51. Xu Z, Becerra AZ, Aquina CT, et al. Emergent colectomy is independently associated with decreased long-term overall survival in colon cancer patients. J Gastrointest Surg 2017;21:543-53.

52. Zattoni D, Christoforidis D. How best to palliate and treat emergency conditions in geriatric patients with colorectal 
cancer. Eur J Surg Oncol 2020;46:369-78.

53. Askari A, Nachiappan S, Currie A, et al. Who requires emergency surgery for colorectal cancer and can national screening programmes reduce this need? Int J Surg 2017;42:60-8.

54. Lim TZ, Tan KK. Endoscopic stenting in colorectal cancer. J Gastrointest Oncol 2019;10:1171-82.

55. Chen YH, Peng JS. Treatment strategy for gastrointestinal tumor under the outbreak of novel coronavirus pneumonia in China. Zhonghua Wei Chang Wai Ke Za Zhi 2020;23:I-IV.

56. Fiori E, Lamazza A, De Cesare A, et al. Palliative management of malignant rectosigmoidal obstruction. Colostomy vs. endoscopic stenting. A randomized prospective trial. Anti-cancer Res 2004;24:265-8.

57. Foo CC, Poon SHT, Chiu RHY, et al. Is bridge to surgery stenting a safe alternative to emergency surgery in malignant colonic obstruction: a meta-analysis of randomized control trials. Surg Endosc 2019;33:293-302.

58. Feo L, Polcino M, Nash GM. Resection of the Primary Tumor in Stage IV Colorectal Cancer: When Is It Necessary? Surg Clin North Am 2017;97:657-69.

59. Raphaeli T, Menon R. Current Treatment of Lower Gastrointestinal Hemorrhage. Clin Colon Rectal Surg 2012;25:219-27.

60. Zheng M, Ma J, Zhao X, et al. Development and reflection of minimally invasive surgery in post-corona virus disease-2019. Chin J Pract Surg 2020;40:245-8.

61. Chinese Society of Colorectal Surgery, Chinese Society of Surgery, Chinese Medical Association, Colorectal and Anal Surgery Committee, Chinese Research Hospitals Association. Chinese expert consensus on surgical diagnosis and treatment strategies for colorectal cancer patients during novel coronavirus peneumonia epidemic. Chin J Pract Surg 2020;40:241-4.

62. Yu G, Lou Z, Zhang W. Some thoughts on colorectal cancer surgery the COVID-19 outbreak. Chin J Gastrointest Surg 2020. [Epub ahead of print].

63. Wrapp D, Wang N, Corbett KS, et al. Cryo-EM structure of the 2019-nCoV spike in the pre-fusion conformation. Science 2020. [Epub ahead of print].

64. Hoffmann M, Kleine-Weber H, Krüger N, et al. The novel coronavirus 2019 (2019-nCoV) uses the SARScoronavirus receptor ACE2 and the cellular protease TMPRSS2 for entry into target cells. bioRxiv 2020. [Preprint].

65. Camargo SM, Singer D, Makrides V, et al. Tissue- specific amino acid transporter partners ACE2 and collectrin differentially interact with hartnup mutations. Gastroenterology 2009;136:872-82.

66. Chen H, Xuan B, Yan Y, et al. Profiling ACE2 expression in colon tissue of healthy adults and colorectal cancer patients by single-cell transcriptome analysis. medRxiv 2020. [Preprint].

67. Hamming I, Timens W, Bulthuis ML, et al. Tissue distribution of ACE2 protein, the functional receptor for SARS coronavirus. A first step in understanding SARS pathogenesis. J Pathol 2004;203:631-7.

68. Han W, Quan B, Guo Y, et al. The course of clinical diagnosis and treatment of a case infected with coronavirus disease 2019. J Med Virol 2020. [Epub ahead of print].

69. Chen N, Zhou M, Dong X, et al. Epidemiological and clinical characteristics of 99 cases of 2019 novel coronavirus pneumonia in Wuhan, China: a descriptive study. Lancet 2020;395:507-13.

70. Guan W, Liang W, Zhao Y, et al. Comorbidity and its impact on 1,590 patients with COVID-19 in China: A Nationwide Analysis. medRxiv 2020. [Preprint].

71. Pile JC. Evaluating postoperative fever: a focused approach. Cleve Clin J Med 2006;73:S62-6.

72. Chen JS, Changchien CR, Tang R. Postoperative fever and survival in patients after open resection for colorectal cancer: a long-term follow-up study of 2,311 prospectively en-rolled patients. Dis Colon Rectum 2008;51:1649-55.

73. Maday KR, Hurt JB, Harrelson P, et al. Evaluating postoperative fever. JAAPA 2016;29:23-8.

74. Mitchell JD, Grocott HP, Phillips-Bute B, et al. Cytokine secretion after cardiac surgery and its relationship to postoperative fever. Cytokine 2007;38:37-42.

75. Barie PS. Surgical site infections: epidemiology and prevention. Surg Infect (Larchmt) 2002;3 Suppl 1:S9-21.

76. Hidron AI, Edwards JR, Patel J, et al. National Healthcare Safety Network Team. NHSN annual update: antimicrobial-resistant pathogens associated with healthcare-associated infections: annual summary of data reported to the National Healthcare Safety Network at the Centers for Disease Control and Prevention, 2006-2007. Infect Control Hosp Epidemiol 2008;29:996-1011.

Cite this article as: Ren X, Chen B, Hong Y, Liu W, Jiang Q, Yang J, Qian Q, Jiang C. The challenges in colorectal cancer management during COVID-19 epidemic. Ann Transl Med 2020;8(7):498. doi: 10.21037/atm.2020.03.158 